\title{
Vivencias e implicación académica en estudiantes universitarios: adaptación y validación de escalas para su evaluación*
}

College students' academic experiences and involvement: assessment scales adaptation and validation

Vivências e envolvimento acadêmico de estudantes universitários: adaptação e validação de escalas para a sua avaliação

\section{Rubén Abello Riquelme, Alejandro Díaz Mujica, María Victoria Pérez Villalobos, Lean- dro S. Almeida, Irma Lagos Herrera, Jaime González Puentes, Brandee Strickland ${ }^{\text {a }}$}

a'Universidad de Concepción, Campus Los Ángeles. Juan Antonio Coloma 0201, Los Ángeles. Telf.: 056 043 - 405218. Correo electrónico: rubenabello@udec.cl

\begin{abstract}
RESUMEN
Este artículo describe los procesos preliminares de adaptación y validación de dos instrumentos diseñados para describir Vivencias Académicas (centradas en la satisfacción) y la Implicación Académica (centrada en el compromiso) en estudiantes universitarios. De acuerdo con la literatura científica en el área, las vivencias y la implicación académica se encuentran asociadas con las dificultades relacionadas al abandono de los alumnos. Para este estudio se aplicaron dos cuestionarios a 261 estudiantes pertenecientes a la Universidad de Concepción, Campus Los Ángeles, Chile. Ambos instrumentos mostraron niveles adecuados de homogeneidad o consistencia interna en los ítems repartidos para las subescalas de cada cuestionario. Paralelamente, se calcularon las correlaciones entre las dimensiones de ambos cuestionarios, siendo los coeficientes obtenidos adecuados para los constructos evaluados. Estos antecedentes permiten el comienzo de la validación de los cuestionarios en estudio.
\end{abstract}

Palabras clave: vivencias académicas, envolvimiento académico, educación superior.

\begin{abstract}
This paper presents preliminary findings regarding the adaptation and validation of two instruments designed to measure Academic Experiences (centred on satisfaction) and Academic Involvement in university students. Previous research has shown that academic experience and involvement are strongly correlated with student drop-out rates. For the present study, the two surveys were administered to 261 students at the University of Concepción, Chile, on the Los Ángeles campus. Both instruments showed suitable levels of reliability and internal consistency on the subscales. Furthermore, the correlation coefficients obtained when comparing dimensions on each survey were acceptable for the constructs being evaluated. These findings allow for a preliminary validation of the two questionnaires included in the study.
\end{abstract}

Key words: academic experiences, academic involvement, higher education.

\section{RESUMO}

Descrevem-se os processos preliminares de adaptação e validação de dois instrumentos voltados para a avaliação das Vivências Acadêmicas (centradas na satisfação) e da Implicação Acadêmica (centrada no compromisso) de estudantes universitários. Conforme a literatura científica na área, vivências e implicação acadêmica encontram-se associadas às dificuldades relacionadas ao abandono dos alunos. Este estudo envolveu a aplicação dos dois questionários a 261 estudantes da Universidade de Concepción, campos Los Angeles, Chile. Ambos os instrumentos mostram níveis adequados

* Esta investigación fue parte del proyecto de Investigación Fondecyt 1040622, "Mecanismos de riesgo y protectores del abandono escolar”; dirigido por el Dr. Alejandro Díaz Mujica de la Universidad de Concepción. 
de homogeneidade ou consistência interna dos itens divididos em subescalas, em cada questionário. Paralelamente, calculam-se as correlações entre as dimensões dos questionários, sendo os coeficientes obtidos adequados aos constructos avaliados. Estes antecedentes permitem o início da validação dos questionários em estudo.

Palavras chave: vivências acadêmicas, envolvimento acadêmico, ensino superior.

\section{INTRODUCCIÓN}

El abandono estudiantil es un fenómeno que preocupa a nivel nacional e internacional en los distintos niveles educacionales. En enseñanza universitaria, aspecto que aquí nos ocupa, existen diversas investigaciones preocupadas en saber las razones intervinientes (Himmel, 2002; Brea de Cabra, 2005; González, Uribe \& González, 2005; Almeida, 2007). Desde una perspectiva socio-política, surge la necesidad de orientar el foco de atención en los aspectos que ayudaron al estudiante a permanecer en la universidad y completar sus estudios pues hay un costo social, familiar e individual detrás de cada alumno que abandona la enseñanza superior o que no concluye sus estudios dentro del número de años apropiado (Almeida, 2007).

En la presente investigación se exploran las vivencias académicas en una lógica de satisfacción y el comportamiento académico en la perspectiva del involucramiento o compromiso de los estudiantes universitarios con sus aprendizajes. Nuestra intención futura es analizar el impacto de algunas variables propias del alumno, así como variables vinculadas a las interacciones con el contexto académico (relaciones interpersonales y relaciones con la institución). En este momento preliminar del proyecto nos interesa analizar la confiabilidad para Chile de dos cuestionarios portugueses en el área de satisfacción y compromiso académico (Almeida, Ferreira \& Soares, 1999; Soares, Almeida, Diniz \& Guisande, 2006).

\subsection{COMPLETACIÓN Y ABANDONO DE ESTUDIOS UNIVERSITARIOS}

Desde los inicios de la Reforma Educacional en Chile, la inversión en nueva infraestructura educacional, los nuevos programas y la política educacional implementada han significado un considerable aumento de la cobertura y del incremento sostenido de la permanencia escolar, especialmente en los niveles de enseñanza básica, y a una mejora considerable en la enseñanza media, que bordea el 90\% (Sapelli \& Torche, 2004). Este cambio implica una población estudiantil terciaria de mayor diversidad, asociada al aumento del abandono de estudios, el que se ha convertido en un fenómeno común en la mayoría de los países latinoamericanos y constituye uno de los problemas prioritarios de investigación (Brea de Cabra, 2005), que reclama acciones adecuadas mitigadoras o de prevención.

En Chile se constata que alrededor del $50 \%$ de quienes ingresan a la universidad no logra completar sus estudios, porcentaje mayor aún en las universidades que no exigen requisitos de ingreso, tales como un determinado puntaje en la prueba de selección universitaria (PSU). Las universidades tradicionales tienen un $73 \%$ de retención, mientras que esta cifra desciende en las universidades privadas a un $65 \%$, y al $52 \%$ en los institutos profesionales (Consejo Superior de Educación, 2006). El porcentaje de abandono es más crítico en áreas como humanidades y derecho, con $80 \%$, y menor en educación y salud, con $37 \%$ y $27 \%$ respectivamente (González et al., 2005). Antes de avanzar importa 
clarificar qué entendemos deserción de los estudiantes de una carrera universitaria como el abandono de los estudios antes de alcanzar el título o grado, considerando un tiempo suficientemente largo para descartar que se trate de una suspensión temporal (González et al., 2005). Un estudiante deviene en desertor cuando abandona voluntariamente, durante tres períodos consecutivos, toda actividad académica programada por la institución de educación superior en la cual se encuentra matriculado; a su vez, la permanencia considera al estudiante que mantiene sus estudios, que no se retira del programa en que se encuentra y lo completa dentro del periodo estimado (Bean \& Eaton, 2002).

La permanencia y la completación de estudios se pueden asociar a rasgos de personalidad como la tolerancia a la frustración y persistencia. En forma paralela, son personas que asisten y trabajan regularmente en las instalaciones que su casa de estudios les ofrece, confían en sus capacidades y desarrollan metas hacia las que se dirigen con su trabajo (Bean \& Eaton, 2002). Así, la completación y la deserción en enseñanza superior han sido objeto de análisis desde la influencia de múltiples variables de tipo personal y de tipo institucional (Tinto, 1975, 1993; Almeida et al., 1999; Bean \& Eaton, 2002; Himmel, 2002). En este artículo nos centramos en algunas características psicológicas de los alumnos, por ejemplo, su compromiso en el contexto académico, su adaptación al curso y sus relaciones interpersonales con los compañeros y los profesores.

Las relaciones interpersonales juegan un importante papel en la decisión de los alumnos de permanecer o abandonar el curso en la institución de enseñanza superior. Se entiende por las relaciones interpersonales los procesos cognitivos, las conductas y los sentimientos experimentados por el estudiante que se encuentra inserto en un contexto social compuesto por compañeros de curso, actividades universitarias de diversa índole e interacciones con sus docentes, que junto con enseñar, proporcionan apoyo a sus estudiantes. Las relaciones interpersonales positivas se caracterizan por sentimientos de pertenencia, de reconocimiento y de bienestar, vinculando los individuos a los contextos en que tales relaciones ocurren (Almeida et al., 1999).

Junto con las relaciones interpersonales, está la universidad como institución que puede ofrecer condiciones favorables tales como servicios estudiantiles, infraestructura, en definitiva, ser un espacio o contexto que le ofrezca al estudiante oportunidad de preparar sus tareas, pasar parte de su tiempo de ocio en las mismas instalaciones y brindar ayuda oportuna a aquellos que tienen carencias de tipo emocional y/o económicas (Almeida et al., 1999; Bean \& Eaton, 2002; Himmel, 2002).

\subsection{ENFOQUES DE ANÁLISIS DE LA RETENCIÓN Y LA DESERCIÓN}

Los enfoques que permiten analizar la retención y la deserción pueden ser agrupados en cinco categorías: psicológico, económico, sociológico, organizacional y de interacciones; además del enfoque integrado, surgido en los últimos años y que utiliza aportes desde las perspectivas ya mencionadas (Himmel, 2002). Del énfasis dado a las variables explicativas, se analiza el problema desde los niveles individual, institucional y familiar.

El enfoque psicológico permite identificar rasgos de personalidad que diferencian a los alumnos que completan sus estudios, versus aquellos que no lo logran; incorpora principalmente variables individuales como características y atributos del estudiante (Himmel, 2002). En primer lugar, es posible sugerir que las intenciones de una persona son producto de sus creencias, estas influyen en sus actitudes y la llevan a manifestar determinados 
comportamientos. Abandonar o continuar en un programa de estudios se ve influido por conductas previas, actitudes acerca de la deserción o persistencia y normas subjetivas acerca de estas acciones, que producen una intención conductual y percepciones acerca de la consecuencia de esta conducta (Himmel, 2002). Al mismo tiempo, la persistencia y la deserción se ven influidas por las percepciones y análisis que hacen los estudiantes de su vida universitaria. Finalmente se expone un modelo basado en los aspectos que han sido incorporados en el presente trabajo y se ha agregado una teoría más general sobre las conductas de logro, las que comprenden atributos como la perseverancia, la elección y el desempeño. Además, se incluye la perspectiva que permite considerar al rendimiento académico previo como una influencia para el desempeño futuro, que actúa sobre el autoconcepto, la percepción de dificultades para estudiar, metas, valores y expectativas de éxito (Himmel, 2002; Díaz, 2004).

Sobre la base de las variables del enfoque psicológico, es posible identificar al joven que ha tenido éxito, a pesar de la adversidad. La investigación indica que las experiencias riesgosas presentes en sus vidas se asocian con factores protectores internos y externos que contribuyen a la resiliencia. Entre éstos es posible destacar un temperamento positivo, creencia en sus habilidades, disponibilidad de oportunidades y recursos para aprovecharlas, modelos y mentores que proporcionan el acceso a la información y muestran opciones para el futuro, suporte social de adultos y participación en grupos pro-sociales, entre otras (Merdirger, Lemon \& Wyatt, 2005). A su vez, y para la problemática del abandono, existen características internas asociadas con el bienestar psicológico, como los niveles de dedicación, auto-eficacia académica y satisfacción con los estudios, que importa considerar pues surgen en la investigación como factores protectores o favorables a la permanencia de los alumnos (Almeida et al., 1999; Salanova, Martínez, Bresó, Llorens \& Grau, 2005).

Es necesario también considerar que los factores de tipo social y organizacional dependen de cómo el individuo los interpreta. Siendo cierto que, en parte, el abandono o la permanencia están afectados por las percepciones de los alumnos sobre el clima y la cualidad de los contextos; de nuevo ciertas características personales de los alumnos son importantes pues determinan sus percepciones. Nos referimos, por ejemplo, a características psicosociales como la eficacia personal, capacidad de adaptación, atribuciones causales, bienestar psicológico, niveles de dedicación, satisfacción con los estudios y estrategias de enfrentamiento al stress. Todas ellas ayudan a comprender las vivencias académicas de los estudiantes en la universidad y como tal son decisivas para el problema que se toma en este estudio (Almeida et al., 1999; Almeida, Gonçalves, Salgueira, Soares, Machado, Fernández, Machado \& Vasconcelos, 2002; Bean \& Eaton, 2002; Himmel, 2002; Brea de Cabra, 2005; Salanova et al., 2005; Almeida, 2007).

El enfoque de las relaciones sociales sugiere que el abandono corresponde a la falta de integración de los estudiantes en el entorno de la Educación Superior (Himmel, 2002). En este sentido, el soporte social proporcionado a través de las relaciones con familiares, colegas y profesores es un importante factor de ayuda al alumno en el enfrentamiento de los desafíos y dificultades en su adaptación académica. Así, contar con un familiar, amigo o profesional que proporcione consejo cuando el estudiante necesita ayuda, es un aspecto a considerar. Paralelamente, y porque no siempre hay el suporte social suficiente, se hace necesaria la existencia de servicios médicos, sociales y de asistencia psicológica (Merdirger et al., 2005). 
Existe evidencia acerca de la influencia de las relaciones del estudiante con la facultad y sus académicos con las actitudes de permanencia y completación de los estudios superiores (Himmel, 2002; Peltier, Laden \& Matranga, 1999). También hay una conexión significativa entre la cultura organizacional y la persistencia del estudiante. El medio ambiente o clima organizacional puede producir un impacto en los niveles de satisfacción, en tanto conecta al alumno con otros estudiantes que se sienten bienvenidos, informados y consistentemente vinculados con su facultad. En efecto, la investigación ha permitido apreciar la importancia del rol social y de la integración académica, respecto a la persistencia del estudiante y su desarrollo académico (Washington \& McKay, 2005).

El desarrollo social y las relaciones interpersonales pueden ejercer influencia favorable en el desarrollo académico y persistencia del estudiante en su esfuerzo por completar su carrera. Los jóvenes, a partir del soporte proporcionado por su familia, obtienen seguridad afectiva y social, al igual que en sus relaciones con académicos y en las diversas experiencias sociales durante sus años de universidad, lo que facilita su permanencia en una carrera (Tinto, 1975, 1993; Peltier et al., 1999; Himmel, 2002; Merdirger et al., 2005; Washington \& McKay, 2005). En el presente estudio se incorporan las relaciones interpersonales, el apoyo personal obtenido, la implicación en los estudios y la adaptación social (Almeida et al., 1999; Almeida et al., 2002).

El enfoque organizacional estudia las características de la institución de Educación Superior, centran su atención en los servicios ofrecidos a los estudiantes y en el clima académico. En este enfoque, se releva la calidad de la docencia, la experiencia de los estudiantes en el aula, los beneficios de salud, actividades deportivas y culturales, los apoyos académicos proporcionados por la organización, los recursos bibliográficos, laboratorios, la cantidad de alumnos por profesor o la calidad arquitectónica y regiones verdes del campus (Tinto, 1975, 1993; Himmel, 2002). Esto significa que es posible abordar el fenómeno de la completación de los estudios, desde el punto de vista de la responsabilidad social de la institución. De modo que a la hora de abordar el tema es necesario considerar los roles, cultura, responsabilidad y estructura de la organización (Washington \& McKay, 2005).

Desde un punto de vista organizacional, es posible observar los recursos que la universidad ofrece y que el estudiante utiliza, los que hacen referencia a la existencia de información acerca de ayuda dirigida al estudiante, el acceso al apoyo económico, las opciones de trabajo remunerado y las actividades complementarias a las académicas o de esparcimiento que puedan llevarse a cabo y la infraestructura que la universidad ofrece (Almeida et al., 1999; Almeida et al., 2002; Merdirger et al., 2005). La carrera universitaria se puede convertir en atractiva en la medida que ofrezca condiciones, tales como estabilidad educativa, satisfacción de las expectativas y objetivos de los estudiantes, un plan de estudios desafiante y la presencia de modelos entre los que están los profesores (Almeida et al., 1999; Merdirger et al., 2005).

La motivación del estudiante se puede ver incrementada en la medida que la universidad ofrezca espacios y actividades complementarias a las propiamente formales que aportan al desarrollo integral del universitario. De igual modo, la satisfacción del estudiante se asocia a la capacidad de la institución para corresponder a las expectativas y proyectos de sus alumnos. Estas condiciones pueden actuar como estimulantes de la disposición del estudiante a hacer tareas académicas aun sin la condición de obligatoriedad que tienen las actividades corrientes (Almeida et al., 2002). Desde la perspectiva 
del enfoque organizacional, el presente estudio incluye las variables valoración de la carrera, adaptación a la institución y utilización de los recursos ofrecidos por la institución (Almeida et al., 1999; 2002).

Con este estudio, se intenta iniciar investigaciones en torno de las vivencias, la adaptación y el suceso académico de estos estudiantes universitarios; nos centramos en un proceso de adaptación y validación preliminar de instrumentos de evaluación de las variables Vivencias Académicas e Implicación Académica. El objeto del estudio se focalizó en la descripción de los resultados en los ítems y en el análisis correlacional de las variables señaladas.

\section{MÉTODO}

\subsection{PARTICIPANTES}

Los sujetos del estudio fueron 261 estudiantes (ver Tabla 1) de dos tipos de carreras universitarias de pregrado: con altos / bajos índices de abandono pertenecientes a una universidad tradicional chilena ubicada en la Región del Bío - Bío. El criterio de selección de la muestra fue de tipo intencional en función de la participación voluntaria, resultando seleccionados estudiantes de Educación Parvularia, Educación Básica, Educación Diferencial, Ingeniería Agroindustrial, Auditoría y Pedagogía en Matemáticas. Es preciso mencionar que en las carreras elegidas se observa una superioridad numérica de mujeres, respecto a varones, esto se reproduce en la composición de la muestra en que 197 son de sexo femenino y 64 masculino.

En la Tabla 1 se observa el número de estudiantes encuestados por carrera con los datos de porcentaje de deserción y puntaje de corte Prueba de Selección Universitaria (PSU). Hay un porcentaje de deserción más elevado en las Carreras de Ingeniería Agroindustrial, Auditoria y Pedagogía en Matemática; en las carreras de Educación General Básica, Educación Parvularia y Educación Diferencial, la deserción es menor y los puntajes de corte observados en la PSU son relativamente menos bajos en las carreras de educación. Sin embargo, las carreras de la muestra no concitan los puntajes más altos de la PSU. La actual muestra requiere ser ampliada para obtener una representación más significativa (ver Tabla 1).

\begin{tabular}{|c|c|c|}
\hline Carrera cursada & $\begin{array}{c}\text { Estudiantes } \\
\text { encuestados }\end{array}$ & $\begin{array}{c}\text { Porcentaje } \\
\text { deserción } \\
\text { año 2006 }\end{array}$ \\
\hline $\begin{array}{c}\text { Ingeniería } \\
\text { Agroindustrial }\end{array}$ & 27 & $35 \%$ \\
\hline Auditoria & 66 & $22,2 \%$ \\
\hline $\begin{array}{c}\text { Pedagogía en } \\
\text { Matemáticas }\end{array}$ & 60 & $21,7 \%$ \\
\hline $\begin{array}{c}\text { Educación General } \\
\text { Básica }\end{array}$ & 33 & $8,9 \%$ \\
\hline Educación Parvularia & 46 & $2,5 \%$ \\
\hline $\begin{array}{c}\text { Educación } \\
\text { Diferencial }\end{array}$ & 29 & $2,5 \%$ \\
\hline Total & 261 & \\
\hline
\end{tabular}

Tabla 1. Caracterización de la muestra.

Carreras y datos del Campus Los Ángeles. Universidad de Concepción 


\subsection{INSTRUMENTOS}

Cuestionario de vivencias académicas (QVA-r) - se destina a evaluar las vivencias y los sentimientos de los universitarios en cuanto a la calidad de las vivencias académicas en el afrontamiento con el contexto universitario (Almeida, Ferreira \& Soares, 1999). Se encuentra diseñado para aplicaciones colectivas, entregándose al sujeto un cuadernillo con preguntas y una hoja de respuestas que debe responder por separado. Cada una de sus alternativas se expresa a través de una escala de formato tipo likert, con cinco posibilidades que van de 1 a 5 , que el sujeto elige para dar a conocer su percepción acerca de lo que se le pregunta (desde 1 - Totalmente en desacuerdo hasta 5 - Totalmente de acuerdo). El cuestionario es compuesto por 60 ítems distribuidos en 5 dimensiones: (Almeida et al., 1999): (1) Dificultades personales, (2) Relaciones interpersonales, (3) Valoración de la carrera, (4) Estrategias de estudio, y (5) Valoración de la institución. El QVA-r es una versión abreviada del QVA que incluye otras dimensiones (Almeida et al., 1999; Almeida et al., 2002).

En lo referente a la validez del instrumento, durante las fases de construcción y validación, el cuestionario ha sido objeto de varias aplicaciones y análisis de tipo cualitativo y cuantitativo de sus ítems; aplicaciones llevadas a cabo en la universidad de Minho, Coimbra y Acores, ubicadas en Portugal. El coeficiente de consistencia interna de las dimensiones siempre se situaron arriba de 0.70 , sumándole a esto validez de contenido, dispersión y poder discriminativo de los ítems, considerados como adecuados (Almeida et $a l .$, 1999). Para el cálculo de la validez de criterio, se realizó una comparación con otras escalas de Autoestima, Solidaridad, Satisfacción con la vida y Ansiedad, obteniéndose datos que se presentaran estadísticamente correlacionados de acuerdo con los constructos evaluados (Almeida et al., 1999).

Cuestionario de Implicación Académica (QEA) - Consta de 38 ítems, evalúa las posibilidades de desarrollo de las expectativas iniciales del estudiante y a lo largo de su curso, reflejando el compromiso de los alumnos en las actividades y contextos académicos. Se encuentra diseñado para aplicaciones colectivas, entregándose al sujeto un cuadernillo con preguntas y una hoja de respuestas que debe responder por separado. Cada una de sus alternativas se expresa a través de una escala de tipo likert, con cuatro posibilidades (de 1-Nunca o casi nunca hasta 4-Siempre o casi siempre). Se evalúan cinco dimensiones: (1) Apoyo personal obtenido, (2) Comportamientos vinculados al desarrollo profesional, (3) Comportamiento social, (4) Utilización de recursos ofrecidos por la institución, y (5) Aprendizaje activo (Abello \& Díaz, 2008; Almeida et al., 1999; Almeida et al., 2002).

\subsection{PROCEDIMIENTOS}

La primera fase de adaptación de los instrumentos QVA-r y QEA, consistió en su traducción desde su idioma original, portugués, al castellano, por parte de dos profesionales bilingües (portugués-español), uno de nacionalidad chilena, un colaborador de los autores del instrumento y finalmente por medio de la revisión de uno de sus creadores, quien hizo llegar modificaciones para algunos de sus reactivos. Se determinó la validez mediante Juicio de Experto (validez de contenido). Participaron docentes y estudiantes del Programa de Magíster en Psicología de la Universidad de Concepción (profesionales del área de la Psicología y de la Pedagogía); estos respondieron un cuestionario en que se les 
solicitó que agruparan los reactivos en cada una de las dimensiones de los instrumentos, de acuerdo a su juicio profesional.

Los instrumentos fueron aplicados en las salas de clases de los respectivos grupos que participaron en forma voluntaria en la investigación, después del previo consentimiento del profesor encargado del curso elegido para ejecutar la aplicación. Durante su desarrollo se encontraba presente un encargado de distribuir los instrumentos y explicar las instrucciones para su contestación, y los profesores, que facilitaron el espacio de sus clases acompañaron el procedimiento. En promedio, los sujetos respondieron en 40 minutos aproximadamente.

La segunda fase consistió en la estimación de Consistencia Interna en cada una de las dimensiones de ambas escalas. Se agruparon los ítems correspondientes a cada sub-escala de los instrumentos Vivencias Académicas (QVA) e Implicación Académica (QEA). Del mismo modo se calculó el coeficiente alpha para cada una de las pruebas considerando todos sus ítems. En una tercera y última fase se agruparon los ítems correspondientes a cada sub-escala de los instrumentos Vivencias Académicas e Implicación Académica y se calculó la mediana, media, desviación estándar, rango de puntuaciones y respectivas intercorrelaciones (coeficiente de correlación producto x momento de Pearson).

\section{RESULTADOS}

En la Tabla 2 se presentan los valores Alfa de Cronbach para cada una de las dimensiones y para el instrumento completo de las vivencias académicas (QVA-r). Como se puede constatar los coeficientes obtenidos son apropiados, oscilan entre 0,68 y 0,85 , y solamente en una dimensión es inferior a 0,70 (generalmente asumido como nivel mínimo exigido). Se presentan los coeficientes de consistencia interna de los ítems (Alfa de Cronbach) para cada una de las dimensiones y en el instrumento completo de envolvimiento académico (QEA). Los coeficientes se situaran entre 0,69 y 0,90, siendo satisfactorios para su futura utilización.

\begin{tabular}{|c|c|}
\hline Sub-escala (dimensión) & Alfa de Cronbach \\
\hline Dificultades personales & 0.82 \\
\hline Relaciones interpersonales & 0.85 \\
\hline Valoración de la carrera & 0.81 \\
\hline Estrategias de estudio & 0.74 \\
\hline Valoración de la institución & 0.68 \\
\hline QVA-R: Escala completa con sus cinco \\
dimensiones & $\mathbf{0 . 7 8}$ \\
\hline Sub-escala (dimensión) & Alfa de Cronbach \\
\hline Apoyo personal obtenido & 0,69 \\
\hline Actividades de la vocación & 0,71 \\
\hline Comportamiento social & 0,80 \\
\hline Recursos ofrecidos por la institución & 0,71 \\
\hline Aprendizaje activo & 0,73 \\
\hline QEA: Escala completa con sus cinco & $\mathbf{0 . 9 0}$ \\
dimensiones & \\
\hline
\end{tabular}

Tabla 2. Alfa de Cronbach en cada una de las dimensiones y en la escala completa Cuestionario de Vivencias Académicas (QVA-R) y Cuestionario de Implicación Académico (QEA) 
En la Tabla 3 es posible ver los valores descriptivos obtenidos para cada una de las dimensiones de las variables Vivencias Académicas e Implicación Académica (ver Tabla 3). Los valores de media obtenidos en Vivencias Académicas (QVA), se situaron arriba del valor 3 que es intermedio en una escala likert de 5 puntos, sugiriendo que la mayoría de los estudiantes presenta opiniones y sentimientos favorables de sus experiencias cotidianas en las relaciones interpersonales, valoran su carrera e institución. Al analizar los valores media obtenidas en Envolvimiento Académico (QEA), se situaron arriba del valor 2,5 que es intermedio en una escala likert de 4 puntos, sugiriendo que los universitarios se encuentran implicados en sus actividades de estudio.

Desde este punto de vista, el promedio de los alumnos no estaría en riesgo de abandonar sus estudios. Sin embargo, al realizar un análisis más detallado en función de la dispersión de los valores individuales en torno de las medias, es posible encontrar siempre un porcentaje de estudiantes, que a pesar de ser minoría, pudiera justificar alguna atención dado sus vivencias menos positivas o frágil envolvimiento académico. Los valores de media más bajos se presentaron en aspectos o dimensiones personales, lo que pudiera justificar alguna atención en futuras pesquisas (ver Tabla 3).

\begin{tabular}{|c|c|c|c|c|c|c|}
\hline $\begin{array}{l}\text { Vivencias } \\
\text { Académicas }\end{array}$ & Mediana & Promedio & $\begin{array}{l}\text { Desviación } \\
\text { estándar }\end{array}$ & Rango & $\begin{array}{l}\text { Puntuación } \\
\text { más alta }\end{array}$ & $\begin{array}{c}\text { Puntuación } \\
\text { más baja }\end{array}$ \\
\hline $\begin{array}{c}\text { Dificultades } \\
\text { personales }\end{array}$ & 2,7 & 2,7 & 0,63 & 3,08 & 4,54 & 1,46 \\
\hline $\begin{array}{c}\text { Relaciones } \\
\text { interpersonales }\end{array}$ & 3,7 & 3,7 & 0.66 & 4 & 5 & 1 \\
\hline $\begin{array}{c}\text { Valoración de la } \\
\text { carrera }\end{array}$ & 3,8 & 3,7 & 0.60 & 2,92 & 4,75 & 1,83 \\
\hline $\begin{array}{l}\text { Estrategias de } \\
\text { estudio }\end{array}$ & 3,5 & 3,5 & 0.45 & 2,67 & 4,87 & 2,20 \\
\hline $\begin{array}{l}\text { Valoración de la } \\
\text { institución }\end{array}$ & 3,3 & 3,2 & 0.69 & 3,57 & 4,86 & 1,29 \\
\hline $\begin{array}{l}\text { Implicación } \\
\text { Académica }\end{array}$ & Mediana & Promedio & $\begin{array}{l}\text { Desviación } \\
\text { estándar }\end{array}$ & Rango & $\begin{array}{c}\text { Puntuación } \\
\text { más alta }\end{array}$ & $\begin{array}{c}\text { Puntuación } \\
\text { más baja }\end{array}$ \\
\hline $\begin{array}{c}\text { Apoyo personal } \\
\text { obtenido }\end{array}$ & 1,9 & 1,9 & 0,46 & 2,63 & 3,63 & 1 \\
\hline $\begin{array}{l}\text { Actividades de } \\
\text { la vocación }\end{array}$ & 2,3 & 2,3 & 0,49 & 2,50 & 3,50 & 1 \\
\hline $\begin{array}{c}\text { Comportamiento } \\
\text { social }\end{array}$ & 2,6 & 2,6 & 0,59 & 2,75 & 4 & 1,25 \\
\hline $\begin{array}{c}\text { Recursos } \\
\text { institucionales }\end{array}$ & 3,1 & 3,1 & 0,54 & 2,57 & 4 & 1,43 \\
\hline $\begin{array}{l}\text { Aprendizaje } \\
\text { activo }\end{array}$ & 2,7 & 2,7 & 0,50 & 1,29 & 3,86 & 2,57 \\
\hline
\end{tabular}

En la Tabla 4, se observa las correlaciones obtenidas entre las dimensiones que forman parte de las variables evaluadas como los dos cuestionarios (Vivencias Académicas e Implicación Académica). Los coeficientes fueran calculados a través del procedimiento producto x momento de Pearson. A partir del análisis de la información presentada, es posible desarrollar algunas inferencias que destacan a continuación y que finalmente se detallan en la Tabla 4. 
Cuando se observa una relación positiva entre las vivencias y la implicación académica, es posible destacar que los estudiantes se identifican y comprometen con la carrera elegida, adoptan perspectivas de desarrollo profesional acordes con sus aptitudes; esto también se relaciona positivamente con su capacidad para estudiar, hábitos de trabajo, gestión del tiempo, utilización de recursos universitarios, infraestructura y los servicios que ella ofrece. Los aspectos mencionados anteriormente también se ven favorecidos en la medida que los estudiantes reciben apoyo en aspectos vocacionales y personales. A medida que los estudiantes participan en forma voluntaria en actividades académicas y de la carrera, también se pueden ver favorecidas la participación en actividades sociales e interacciones con sus profesores.

Por el contrario, en la medida que los estudiantes experimentan angustia, desorientación, soledad, debilidad física, pesimismo, inestabilidad emocional y tristeza, es posible que el alumno percibirá una disminución en el soporte social que proporcionan las relaciones sociales con pares y académicos. Esta disminución también se extiende a los aspectos organizacionales como la calidad de la docencia, plan de estudios desafiante y el uso de los recursos que la universidad ofrece (ver Tabla 4).

\begin{tabular}{|c|c|c|c|c|c|c|c|c|c|}
\hline & $\begin{array}{l}\text { Difinuth des } \\
\text { persorndes }\end{array}$ & $\begin{array}{c}\text { Relaciores } \\
\text { interpersorales }\end{array}$ & $\begin{array}{l}\text { Wharación } \\
\text { de la carrera }\end{array}$ & $\begin{array}{l}\text { Eitrategis } \\
\text { de estudio }\end{array}$ & $\begin{array}{c}\text { Whloxión } \\
\text { de hrettoxín }\end{array}$ & $\begin{array}{c}\text { Aporo } \\
\text { persanal } \\
\text { Obterido }\end{array}$ & $\begin{array}{l}\text { Actividades de } \\
\text { ln mocación }\end{array}$ & $\begin{array}{c}\text { Comportamierto } \\
\text { Socinl } \\
\end{array}$ & $\begin{array}{c}\text { Recursos } \\
\text { instimcionales }\end{array}$ \\
\hline Dricultades & ] & & & & & & & & \\
\hline $\begin{array}{c}\text { Relaciones } \\
\text { Intarpersonales }\end{array}$ & $-0.285 * *$ & 1 & & & & & & & \\
\hline $\begin{array}{l}\text { Whación } \\
\text { de la carrera }\end{array}$ & $-0.276 * * *$ & $0.260 * * *$ & 1 & & & & & & \\
\hline $\begin{array}{l}\text { Estrategns } \\
\text { de esturio }\end{array}$ & $-0,331 * * *$ & $0.266^{* * *}$ & $0385^{* * *}$ & 1 & & & & & \\
\hline 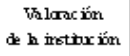 & $-0,195 * *$ & $0,238 * *$ & $0,536^{* * *}$ & $0.286 * * *$ & 1 & & & & \\
\hline $\begin{array}{l}\text { Apoyo personal } \\
\text { Obtanido }\end{array}$ & $-0,080$ & $0.262 * *$ & $0.219 * * *$ & $0,397 * * *$ & $0.214 * * *$ & 1 & & & \\
\hline $\begin{array}{l}\text { Actividades de } \\
\text { la wocación }\end{array}$ & $-0.216 * *$ & $0,396 * *$ & $0,449^{* * *}$ & $0,400 * *$ & $0,320^{* * *}$ & $0,599 * * *$ & 1 & & \\
\hline $\begin{array}{l}\text { Compathmierto } \\
\text { Socis } 1\end{array}$ & $-0,091$ & $0,502 * * *$ & $0,212 * *$ & $0,221 * * *$ & $0,135^{*}$ & $0,445 * \cdots$ & $0,6366^{* * *}$ & 1 & \\
\hline $\begin{array}{l}\text { Recursos } \\
\text { Irotibucionsles }\end{array}$ & $-0,080$ & $0.266 * *$ & $0.306^{* * *}$ & $0,310 * *$ & $0364 * * *$ & $0,352 * * *$ & $0,409 * * *$ & $0,368 * * *$ & 1 \\
\hline $\begin{array}{c}\text { Arrerdimje } \\
\text { Activo }\end{array}$ & $-0,101$ & $0.254^{* * *}$ & $0,32 \mathrm{~S}^{* * *}$ & $0,544 * * *$ & $0,221 * * *$ & $0,547 * * *$ & $0,578 *+*$ & $0,472 * * *$ & $0,403 * * *$ \\
\hline
\end{tabular}

Tabla 4. Relación entre comportamiento académico y vivencias académicas Leyenda: Sin asterisco, significa que el valor obtenido no es significativo $* p<.05 ; * * p<.01 ; * * * p<.001$ 


\section{CONCLUSIONES}

Debido a la multiplicidad de aspectos que se pueden considerar en la análisis del proceso de adaptación y rendimiento académico de los alumnos en la universidad, y a la relevancia de los procesos para el abandono o permanencia hasta la conclusión exitosa de sus cursos, optamos en este proyecto de pesquisa por considerar dos instrumentos de medida capaces de abarcar algunos de estos factores que nos parecen más relevantes. Así, tomamos un cuestionario centrado en las vivencias académicas y otro dirigido a la implicación académica de alumnos en enseñanza superior.

En este estudio exploramos las características psicométricas iniciales del Cuestionario de Vivencias Académicas (QVA-r) y del Cuestionario de Implicación Académica (QEA). Los coeficientes obtenidos en torno de la dispersión de los resultados en los ítems, así como los coeficientes de la consistencia interna de las sucesivas dimensiones de cada cuestionario en las correlaciones entre las dimensiones de los dos cuestionarios nos afirman sus propiedades métricas en términos de precisión y de validez.

Tomando los puntajes obtenidos por los ítems y dimensiones en este primer estudio, se verifica alguna convergencia de factores institucionales y personales, asumidos como muy importantes en la toma de decisión de permanecer o abandonar la universidad (Almeida et al., 1999; Almeida et al., 2002; Bean \& Eaton, 2002; Brea de Cabra, 2005; Himmel, 2002; Lohfink \& Paulsen, 2005; Tinto, 1975, 1993). En forma paralela, las relaciones sociales con los servicios, los agentes académicos y las vivencias sociales se pueden entender como un importante factor de pertenencia a la institución y de ayuda cuando sea necesario. Por norma, un estudiante satisfecho con la vida social y/o que participa como ayudante académico, aumenta la probabilidad de persistir en la institución que los acoge. De la misma forma, los servicios que la universidad ofrece a los estudiantes, la calidad de la docencia, los recursos bibliográficos, de laboratorio o deportivas y culturales son muy importantes en la decisión de mantener o abandonar un curso de formación y una determinada institución.

Lógicamente que en el futuro nuevos estudios merecen ser realizados para consolidar la validez de los dos cuestionarios y sus dimensiones evaluadas. Una muestra más consistente y representativa de los alumnos necesita ser considerada. Interesa analizar el funcionamiento de las dimensiones de los instrumentos en los alumnos de diferentes titulaciones en las distintas fases de su curso académico (al inicio o al concluir, por ejemplo). También importa analizar cómo estas variables se diferencian o asocian según el género, la edad, el origen social o el estatus de un alumno tradicional, comparado con alumnos más antiguos, con compromisos laborales y familiares. Al mismo tiempo será fundamental disponer de datos longitudinales para apreciar la evolución de las dimensiones a lo largo de la carrera académica de los alumnos y tener medidas de su rendimiento académico y de sus decisiones de permanecer o abandonar como forma de apreciar la capacidad predictiva de las diversas dimensiones evaluadas en estos comportamientos académicos que tanto inquietan hoy a las universidades, las familias y la sociedad en general.

Al terminar, comprendemos que hacer un análisis empírico a los enfoques psicológico, de redes sociales y organizacional, incluye diversas variables que necesitan ser estudiadas por medio de distintos instrumentos y procedimientos más completos que los que aquí se presentan, por este motivo, a pesar de que se expone un avance en el estudio de esta materia, éste también presenta limitaciones; importa dejar explícito que el alcance del 
trabajo presentado se enfoca en los instrumentos que han sido utilizados y descritos en el cuerpo de esta publicación.

\section{REFERENCIAS BIBLIOGRÁFICAS}

Abello, R. \& Díaz, A. (2008). Vivencias, comportamiento académico, atribuciones causales, metas de estudio y autoconcepto en estudiantes universitarios. (Tesis para optar al título de Magíster). Concepción: Universidad de Concepción, Escuela de Graduados.

Almeida, L. S. (2007). Transição, adaptação académica e êxito escolar no ensino superior. Revista Galego-Portuguesa de Psicoloxía e Educación, 14 (2), 203-215.

Almeida, L., Gonçalves, A., Salgueira, A., Soares, A., Machado, C., Fernandes, E., Machado, J. \& Vasconcelos, R. (2002). Expectativas de envolvimento académico à entrada na universidade: Estudo com alunos da Universidade do Minho. Minho: Universidade do Minho.

Almeida, L., Ferreira, J. \& Soares, A. (1999). Questionário de vivências académicas (QVA e QVAr). Minho: Universidade do Minho \& Universidade de Coimbra.

Bean, J. \& Eaton, S. (2002). The psychology underlying successful retention practices. Journal of College Student Retention, vol.3, n.1, 73-89.

Brea de Cabra, M. (2005). Deserción en la educación superior pública en República Dominicana. Psicologíacientifica.com. Recuperado el 10 de mayo, 2006 de http://www.psicologiacientifica. com

Díaz, A. (2004). Procesos cognitivo motivacionales y calificaciones académicas. (Memoria para optar al título de Magíster). Concepción: Universidad de Concepción, Escuela de Graduados.

Estudio Índices 2007. (2006, Noviembre 9). El Mercurio. P. C7.

González, L., Uribe, D. \& González, S. (2005). Estudio sobre la repitencia y la deserción en la educación superior chilena (IESALC / IESALC National Reports). Santiago: IESALC UNESCO. Extraído el 05 de junio, 2006, de http://www.iesalc.unesco.org.

Himmel, E. (2002). Modelos de análisis de la deserción estudiantil en la educación superior (Consejo Superior de Educación). Santiago: República de Chile. Extraído el 01 de junio 2006, de http:// www.cse.cl/Asp/WEB_CSEpublic_pdf.asp

Lohfink, M. \& Paulsen, M. (2005). Comparing the determinats of persistence for first - generation and continuing - generation students. Journal of College Student Development, vol. 46, n.4, $409-428$.

Merdirger, J., Hines, A., Lemon, K. \& Wyatt, P. (2005). Pathways to college for former foster youth: Understanding factors that contribute to educational success. Child Welfare, vol.6, n. 84, 867-896.

Peltier, G., Laden, R. \& Matranga, M. (1999). Student persistence in college: A review of research. Journal of College Student retention, vol.1, n.4, 357-375.

Salanova, S., Martínez, I., Bresó, E., Llorens, S. \& Grau, R. (2005). Bienestar psicológico en estudiantes universitarios: facilitadores y obstaculizadores del desempeño académico [versión electrónica]. Revista Anales de Psicología, vol.1, n.21, 170-180. Recuperado el 12 de mayo de 2006, en http://www.um.es/analesps/v21/v21_1/16-21_1.pdf

Sapelli, C. \& Torche, A. (2004). Deserción escolar y trabajo juvenil: ¿Dos caras de una misma decisión? Cuadernos de Economía, vol.41, 173-198.

Soares, A., Almeida, L., Diniz, A. \& Guisande, M. (2006). Modelo multidimensional de ajustamento de jovens ao contexto universitário (MMAU). Estudo com estudantes de ciências e tecnologias versus ciências sociais e humanas. Análise Psicológica, vol.1, n.24, 15-27.

Tinto, V. (1975). Dropout from higher education: A theoretical synthesis of recent research. Review of Educational Research, vol.45, n.1 89-125.

Tinto, V. (1993). Leaving College. Chicago: The University of Chicago Press. 

VIVENCIAS E IMPLICACIÓN ACADÉMICA EN ESTUDIANTES UNIVERSITARIOS: ADAPTACIÓN Y VALIDACIÓN...

Washington, E. \& McKay, S. (2005). Strategic planning and retention within the community college setting. College Student Affairs Journal, vol.25, n.1, 50-63. 